\title{
Solute trapping-free massive transformation at absolute stability
}

\author{
A. Jacot*, M. Sumida, W. Kurz \\ Institute of Materials, Swiss Federal Institute of Technology Lausanne, CH-1015 Lausanne EPFL, Switzerland \\ Received 12 October 2010; received in revised form 8 November 2010; accepted 14 November 2010 \\ Available online 14 December 2010
}

\begin{abstract}
Directional transformation of a hypo-peritectic Fe-17.5 at.\% Co alloy was studied. Two consecutive phase transformationssolidification (liquid to delta ferrite) and solid-state transformation ( $\delta$ ferrite to $\gamma$ austenite) — were observed and compared with theory. In all experiments, the solidification front was planar and in the steady-state, and therefore produced a homogeneous parent phase for the following $\delta-\gamma$ transformation. Depending on the growth conditions, $\gamma$ transformed from $\delta$ as cells or as a plane front. The cell tip radius decreased with growth rate from $V=1-5 \mu \mathrm{m} \mathrm{s}^{-1}$. At higher velocities, between 7 and $10 \mu \mathrm{m} \mathrm{s}{ }^{-1}$, the $\delta / \gamma$ interface morphology became planar. In order to explain this morphological transition, volume diffusion-controlled plane front growth and dendrite growth theory was applied. Good agreement was obtained between theory and experiments. It is concluded that plane front stabilization with increasing velocity is due to absolute stability, with a concentration spike at the transformation front. In the steady-state, this leads to composition invariance, typical for massive transformation. Computed interface velocities for quenching in heat treatment, which can be as high as several centimeters per second, show that, in certain cases, the controlling mechanism of massive transformation is steady-state plane front growth with a narrow concentration spike and not complete solute trapping.
\end{abstract}

(C) 2010 Acta Materialia Inc. Published by Elsevier Ltd. All rights reserved.

Keywords: Massive transformation; Local equilibrium; Solute trapping; Absolute stability; Directional transformation

\section{Introduction}

Solid-state transformations are very important in material processing as, in combination with solidification, they largely determine the properties of the final product. Understanding the mechanisms of these transformations is therefore important. One such phase change is the massive transformation characterized by a composition invariant change in the crystal structure with a highly mobile interface. Much work has been published in this area, mostly using isothermal or continuous cooling experiments $[1,2]$. The high displacement rates of this transformation (typically millimeters per second to centimeters per second) make experiments difficult to interpret, owing to the uncertainty in the interface temperature associated with the latent heat release [2]. Directional growth experiments are more interesting, as the interface velocity $V$ and tempera-

\footnotetext{
* Corresponding author. Tel.: +41 21693 3920; fax: +41 216935890. E-mail address: alain.jacot@epfl.ch (A. Jacot).
}

ture gradient $G$ can be set, and the interface temperature $T_{\mathrm{i}}$ measured. Borgenstam and Hillert [3] and Zurob et al. [4], used directional growth experiments in a concentration gradient which allowed them to quantify the $\gamma-\alpha$ transformation in $\mathrm{Fe}-\mathrm{Ni}$ and $\mathrm{Fe}-\mathrm{C}-\mathrm{Mn}$.

Vandyoussefi et al. [5] and Kurz and Lima [6,7] used directional growth in a temperature gradient (Bridgman technique) to study the $\delta-\gamma$ transformation in $\mathrm{Fe}-\mathrm{Ni}$ and $\mathrm{Fe}-\mathrm{Cr}$, respectively. In Fig. 1, the differences between isothermal and directional growth experiments are presented, with the aim of showing the advantage of studying solidstate transformations in a temperature gradient. Fig. 1a represents a schematic drawing of a massive transformation product forming from a grain boundary in the center outwards. In this case, the growth direction and the heat flux point in the same direction. At the interface, the rejection of solute and heat sets the interface temperature, which cannot be measured owing to the small boundary layers. On the other side, a directional growth experiment is characterized by a unidirectional temperature field with 

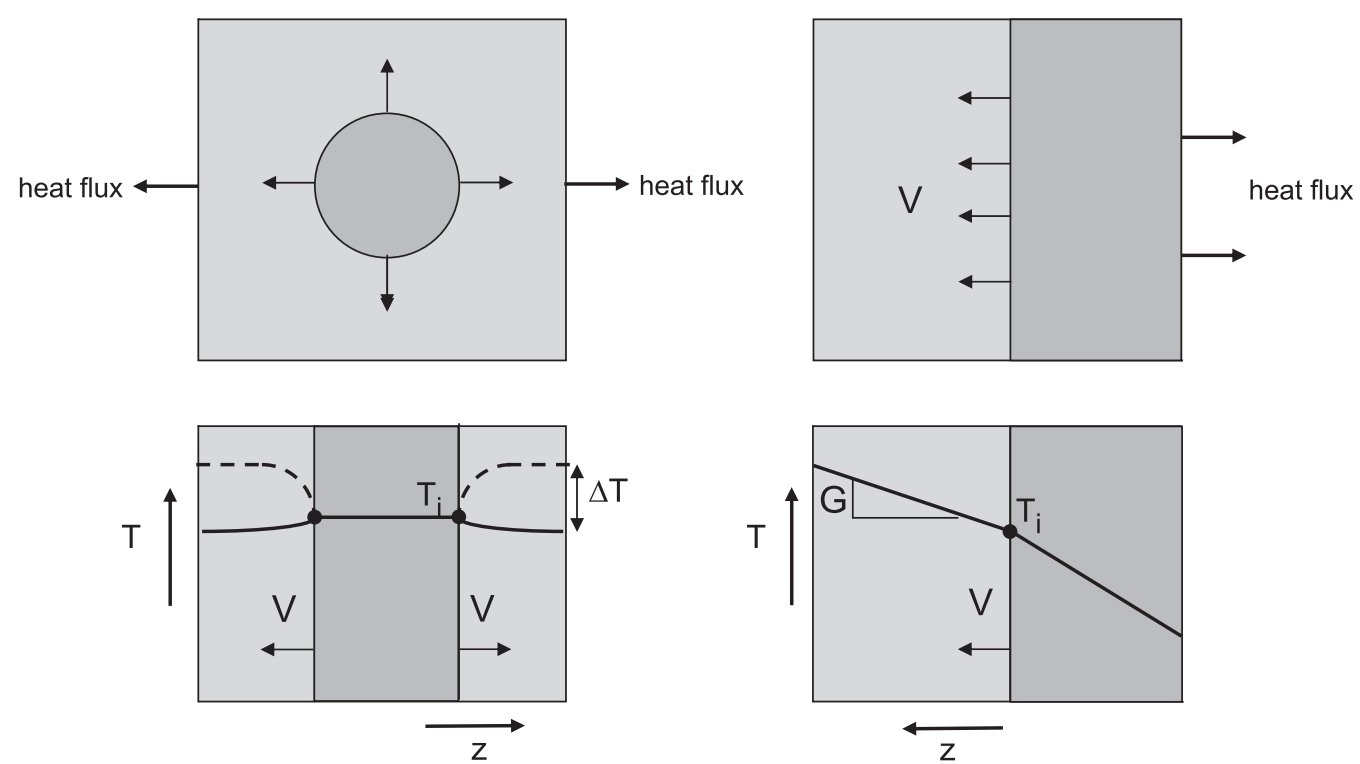

(a)

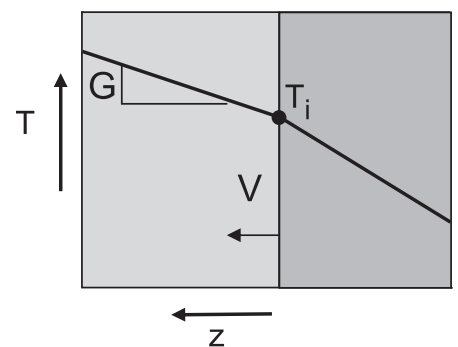

(b)

Fig. 1. Solid-state transformation conditions: (a) isothermal transformation with nucleation in the undercooled bulk and outwards growth of the new (dark) phase as a function of the undercooling $\Delta T$; the rejection of solute and heat defines the interface temperature $T_{\mathrm{i}}$, which cannot be measured; (b) directional transformation with nucleation at the cold outer surface; interface velocity $V$, temperature gradient $G$ and $T_{\mathrm{i}}$ can be measured.

a positive temperature gradient (Fig. 1b). The interface moves here opposite to the heat flux, and its velocity can be set to specific values which are only limited by the condition of unidirectional heat flux. Owing to this configuration, the interface velocity, the temperature gradient and the interface temperature can be easily measured. From this brief presentation of the two techniques, it should become clear that directional growth experiments have a potential for future solid-state transformation studies.

$\mathrm{Fe}$ alloys, which form the basis of the industrially important steels, show a rich variety of solid-state transformations on cooling. This is also the case for peritectic $\mathrm{Fe}-$ Co alloys. On the iron-rich side of this system, bcc $\delta$-ferrite $\left(\alpha_{\text {high T }}\right)$ solidifies on cooling and transforms subsequently to fcc $\gamma$-austenite, which in turn transforms back to $\alpha$-ferrite. The $\gamma-\alpha$ transformation process is affected by the microstructure of austenite formed during the $\delta-\gamma$ transformation. Very little information is available on the latter transformation. This is attributed to the fact that the growth behavior of $\gamma$ is not easy to observe, since it occurs close to the solidus temperature of the alloy, and the microstructure of the $\delta-\gamma$ transformation is masked by the consecutive $\gamma-\alpha$ transformation at lower temperature. Furthermore, owing to the small concentration difference between the phases, metallographic evidence of the transformation product is very difficult to obtain.

Vandyoussefi et al. [5] observed cellular interface morphologies of various shapes and scales in directionally transformed $\mathrm{Fe}-3 \% \mathrm{Ni}$ alloys. According to their findings, the cell spacing decreased when the velocity was increased from 5 to $25 \mu \mathrm{m} \mathrm{s}^{-1}$. At $30 \mu \mathrm{m} \mathrm{s}^{-1}$, it coarsened and showed the typical form of low-amplitude interface deformations. At velocities $>40 \mu \mathrm{m} \mathrm{s}^{-1}$, signs of a transformation front could no longer be detected. It was argued that the boundary layer at the moving interface was too thin, and the concentration spike too small to become visible in the metallographic etching. Kurz and Lima [6,7] observed similar morphological transitions in $\mathrm{Fe}-8 \% \mathrm{Cr}$. In both cases $(\mathrm{Fe}-\mathrm{Ni}$ and $\mathrm{Fe}-\mathrm{Cr})$, the variation in the dendrite/cell spacing $\lambda$ was compared with theoretical predictions of the Ivantsov-Marginal stability (I-MS) model [8,9], and it was concluded that the observations could be explained by the well-known growth theory of dendrites, cells and plane front [10]. The disappearance of the transformation microstructure at high velocity was explained by the concept of absolute stability [11].

At absolute stability, the solute diffusion field at the interface becomes localized with respect to the instability wavelength $\lambda$. Effects of large solute Péclet number (ratio of diffusion coefficient to interface velocity $D / V \ll \lambda$ ) and capillarity dominate, leading to a morphologically stable planar transformation front. (Oscillatory instabilities, which do not form in alloys with very small solidusliquidus intervals, are neglected here [12].) When the transformation produces a plane front in steady-state, the parent phase and the product phase have the same composition.

As the critical velocity required for absolute stability $V_{\mathrm{ab}}$ is proportional to the solute diffusion coefficient, the typical velocity for absolute stability in solidification is very high and very difficult to produce [13-15]. An absolutely stable planar growth front has, to the knowledge of the authors, never been shown very clearly, either in solidification or in solid-state transformation experiments. In solid-state transformations, the limit of absolute stability is orders of magnitude smaller and can be easily reached. 
The objectives of the study were: (i) to produce the first visible evidence of an absolutely stable (planar) interface in solid-state transformation; (ii) to analyze the solid-state transformation microstructures with the aid of solidification models; and (iii) answer the question of whether a massive transformation can be formed by steady-state plane front growth with a composition spike rather than by complete solute trapping (complete loss of local equilibrium).

The latter mechanism was proposed by Hillert in 1969 [21], but it seems that no clear evidence has been given so far. The present study is based on a series of directional transformation experiments using a $\mathrm{Fe}-\mathrm{Co}$ alloy. The results are analyzed using solidification theory.

\section{Experimental}

The $\delta-\gamma$ transformation in hypo-peritectic $\mathrm{Fe}-17.7$ at.\% Co was selected for this study because of its high solvus temperature (Fig. 2a [16]), which is associated with high interface mobility and fast diffusion. The composition was chosen to be close to the maximum solubility of $\delta$ with both transformation fronts (solidification and solid-state transformation) close to each other. As shown in Fig. 2b, the concentration spikes for both transformations are opposite to each other owing to the different distribution coefficients of the peritectic phase diagram.

The vacuum-cast specimens with length $140-200 \mathrm{~mm}$ were placed in alumina tubes $6 \mathrm{~mm}$ in inner diameter. At first, the samples were lowered through a Bridgman apparatus $\left(T_{\max }=1670{ }^{\circ} \mathrm{C}\right)$ at a rate of $2 \mathrm{~mm} \mathrm{~s}^{-1}$ until $30 \mathrm{~mm}$ of the crucible were in the liquid metal cooler (In-Ga-Sn alloy at room temperature). After temperature stabilization of the specimen, the velocity was set to the chosen values in the range where absolute stability was expected, i.e., $1 \leqslant V \leqslant 10 \mu \mathrm{m} \mathrm{s}^{-1}$. At the end of the experiment, the specimen was dropped into the liquid metal bath for quenching to reveal the growth morphology. The growth length of the specimen $25<L_{\mathrm{g}}<55 \mathrm{~mm}$ was longer than the initial transient for solidification (see below). The temperature gradient was measured in a previous experiment with an $\mathrm{Fe}-$ 4 at.\% Ni alloy with similar properties, transformation temperatures and growth rates [17]. The weighted temperature gradient at the growth front was found to be $18 \mathrm{~K} \mathrm{~mm}^{-1}$.

The specimens were cut longitudinally along the cylinder axis and prepared for microscopic examination. Oberhoffer's etch was used to reveal the microstructure. The compositions of the specimens were determined by an electron probe micro-analyzer (EPMA) with a wavelength dispersive

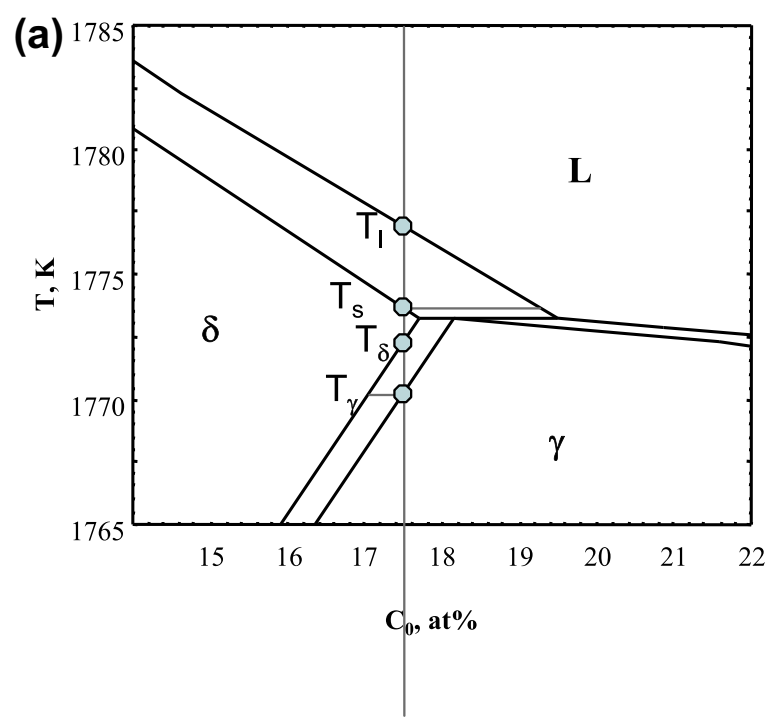

(b)

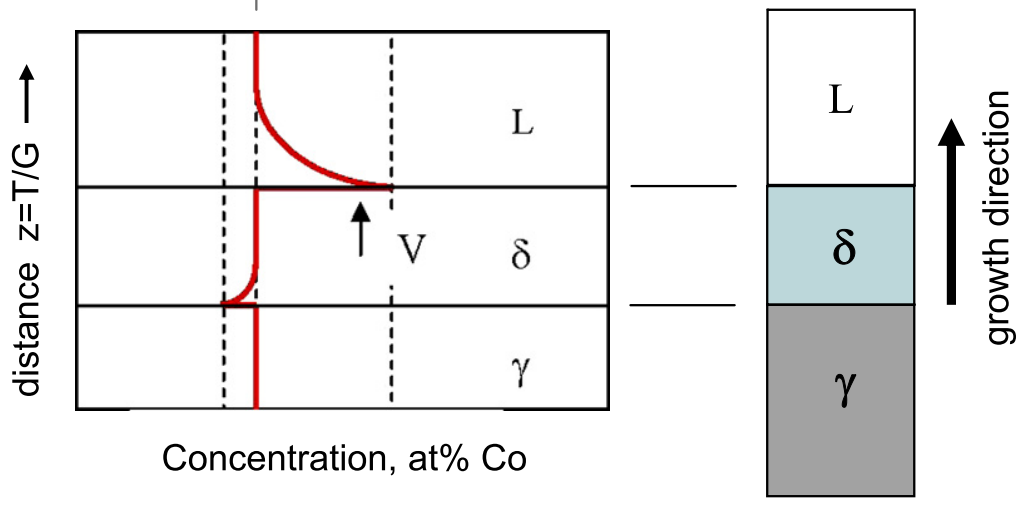

Fig. 2. (a) Phase diagram of $\mathrm{Fe}-\mathrm{Co}$ system in the Fe-rich high-temperature region. (b) Steady-state solute pile up at planar $L / \delta$ and $\delta / \gamma$ interfaces. The boundary layers are opposite to each other, owing to the different distribution coefficients $\left(k_{\delta}<1, k_{\gamma}>1\right)$. 
spectroscope. Typically, 20 spots were analyzed in each region, and the mean value was calculated. Calibration was obtained with pure $\mathrm{Fe}$ and $\mathrm{Co}$.

\section{Results}

Figs. 3 and 4 show micrographs of the $L / \delta$ interface and the $\delta / \gamma$ front. The longitudinal sections show that the $\delta / \gamma$ interface morphology varies with increasing $V$, from coarse deep cells at $V=1 \mu \mathrm{m} \mathrm{s}^{-1}$ (Fig. 3a) to finer low-amplitude cells at $V=5 \mu \mathrm{m} \mathrm{s}^{-1}$ (Fig. 3c), to flat cells at $V=7 \mu \mathrm{m} \mathrm{s}^{-1}$ (Fig. 4a) and finally to a plane front at $V=10 \mu \mathrm{m} \mathrm{s}^{-1}$ (Fig. 4b). The transverse sections show weakly segregating hexagonal cells at $1 \mu \mathrm{m} \mathrm{s}^{-1}$ (Fig. 3b) and platelike cells arranged along rows at $5 \mu \mathrm{m} \mathrm{s}^{-1}$ (Fig. 3d). The microsegre- gation of the flat cells at $V=7 \mu \mathrm{m} \mathrm{s}^{-1}$ (Fig. 4a) was so weak that no trace of microstructure could be found in the transverse section. The cell spacings were determined from transverse sections at 1 and $5 \mu \mathrm{m} \mathrm{s}^{-1}$ (Table 1, Fig. 5). The amplitude $\varepsilon$ of the cells was measured from longitudinal sections (Fig. 5).

Composition profiles across the $\delta / \gamma$ interfaces parallel to the growth direction at the center of the samples and along the center of a cell for $V=1$ and $10 \mu \mathrm{m} \mathrm{s}^{-1}$ were obtained by EPMA in $10-\mu \mathrm{m}$ steps (Fig. 6). At $1 \mu \mathrm{m} \mathrm{s}^{-1}$, the composition of the $\gamma$ cells is slightly higher than that of $\delta$ and a $\sim 35$ - $\mu$ m-thick boundary layer can be seen. The composition of the plane front $\gamma$ at $V=10 \mu \mathrm{m} \mathrm{s}^{-1}$ is the same on both sides of the transformation front. No concentration spike was detected at the higher rate.

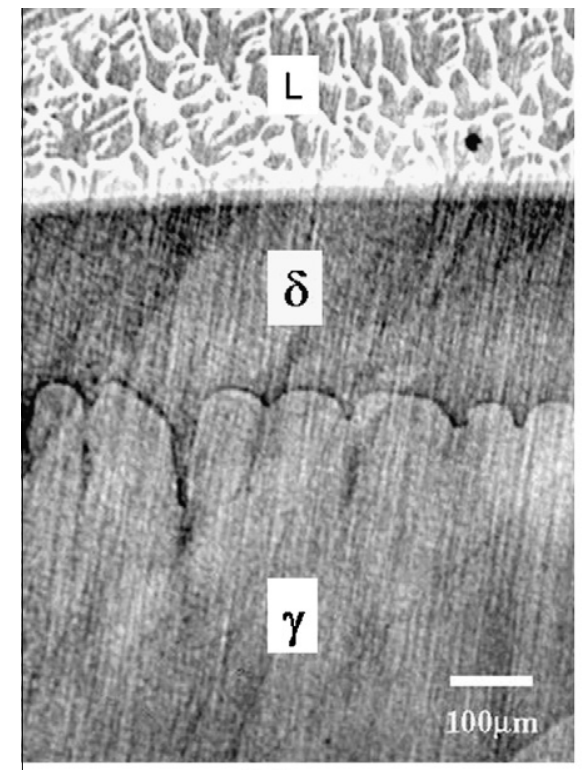

(a)

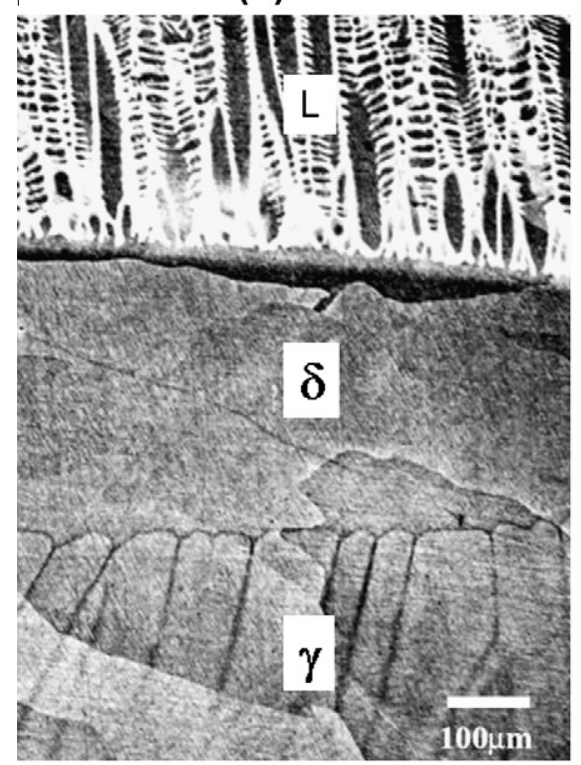

(c)

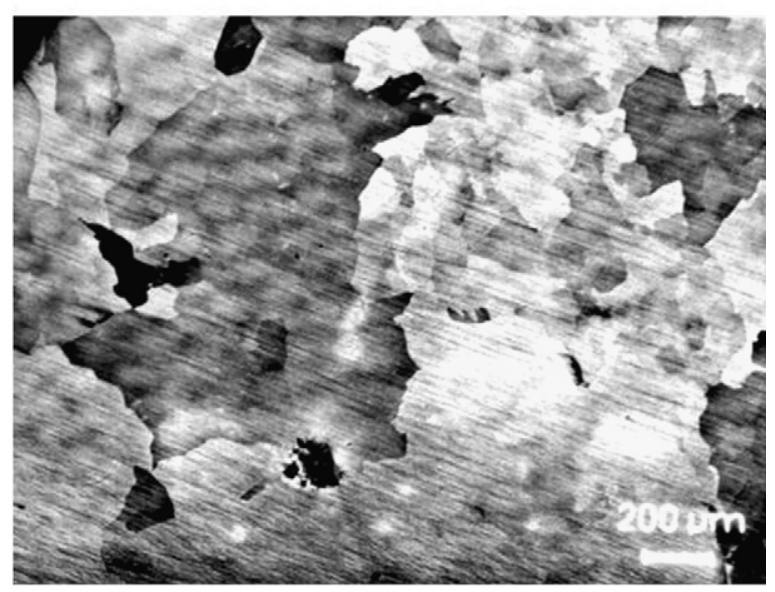

(b)

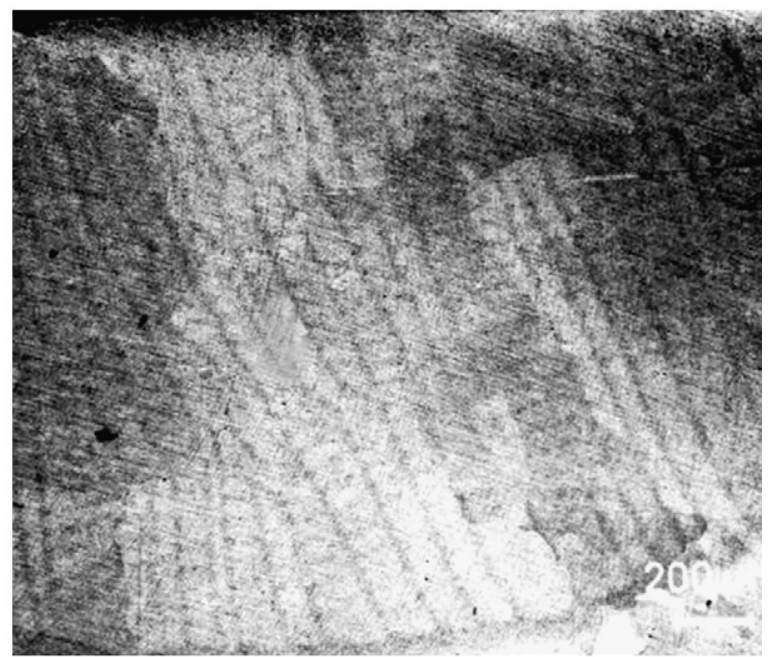

(d)

Fig. 3. Micrographs of longitudinal and transverse sections close to the $L / \delta$ and $\delta / \gamma$ interfaces showing (a and b) coarse cells at $V=1$ and (c and d) shallow cells at $V=5 \mu \mathrm{m} \mathrm{s}^{-1}$. 


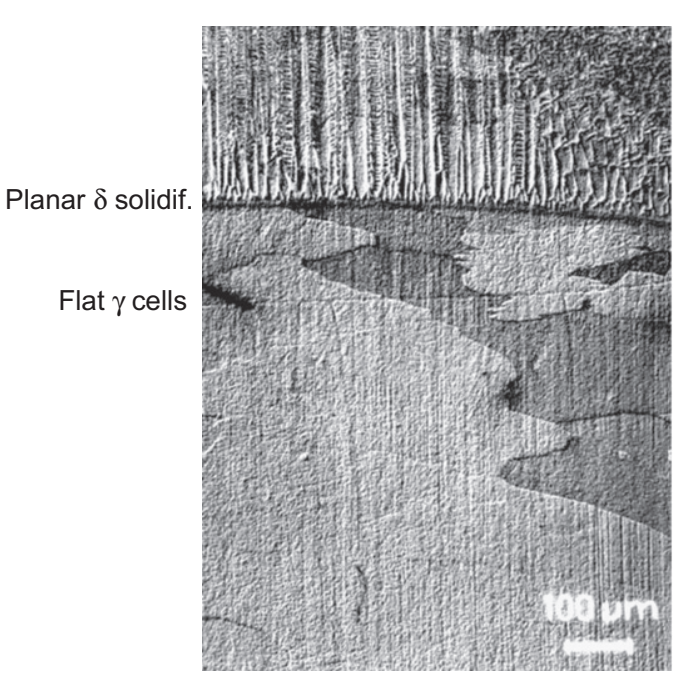

(a)

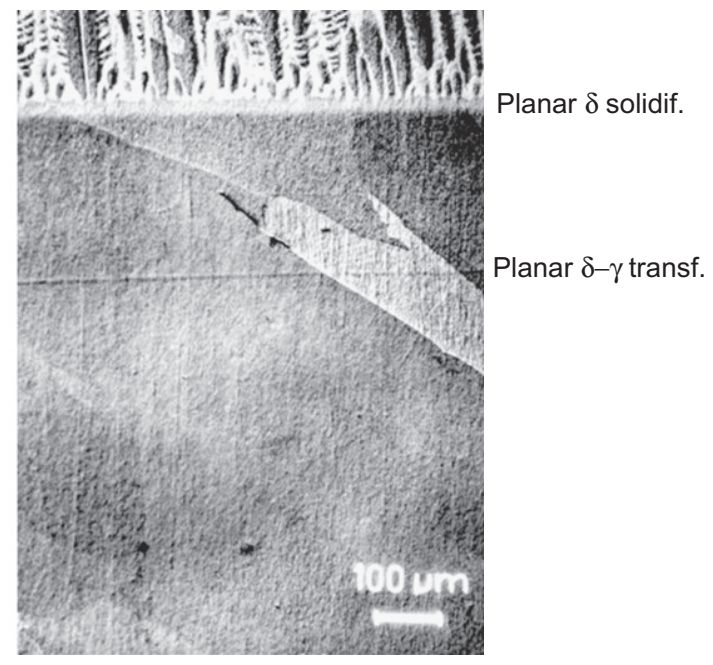

(b)

Fig. 4. Micrographs of longitudinal sections close to the $L / \delta$ and $\delta / \gamma$ interfaces showing: (a) very flat cells at $V=7$ and (b) the plane front at $V=10 \mu \mathrm{m} \mathrm{s}^{-1}$.

Table 1

Characteristic data for solid-state transformation structures $\left(L_{\mathrm{g}}\right.$ : growth length).

\begin{tabular}{llllllllll}
$\begin{array}{l}V \\
\left(\mu \mathrm{m} \mathrm{s}^{-1}\right)\end{array}$ & $L_{\mathrm{g}}(\mathrm{mm})$ & $\begin{array}{l}C_{\mathrm{o}}=C_{\delta} \\
(\mathrm{at} \%)\end{array}$ & $C_{\gamma}(\mathrm{at} . \%)$ & $\begin{array}{l}\lambda(\mathrm{min}) \\
(\mu \mathrm{m})\end{array}$ & $\begin{array}{l}\lambda(\max ) \\
(\mu \mathrm{m})\end{array}$ & $\begin{array}{l}\lambda_{\text {mean }} \\
(\mu \mathrm{m})\end{array}$ & $\begin{array}{l}\varepsilon_{\text {mean }} \\
(\mu \mathrm{m})\end{array}$ & Morphology & Fig. no. \\
\hline 1 & 25 & 17.52 & 17.55 & 95 & 120 & 107 & 104 & $\begin{array}{l}\text { Deep cells } \\
\text { Shallow cells of rectangular }\end{array}$ & $3 \mathrm{c}$ and d \\
5 & 29 & n.d. & n.d. & $55 / 82$ & $63 / 109$ & $59 / 95$ & 17 & $\begin{array}{l}\text { b } \\
\text { section }\end{array}$ \\
7 & 38 & n.d. & n.d. & n.d. & n.d. & n.d. & $\sim 5$ & $\begin{array}{l}\text { Flat cells } \\
\text { Plane front }\end{array}$ \\
10 & 55 & 17.45 & 17.45 & $\infty$ & $\infty$ & $\infty$ & 0 & $4 \mathrm{~b}$ \\
\hline
\end{tabular}

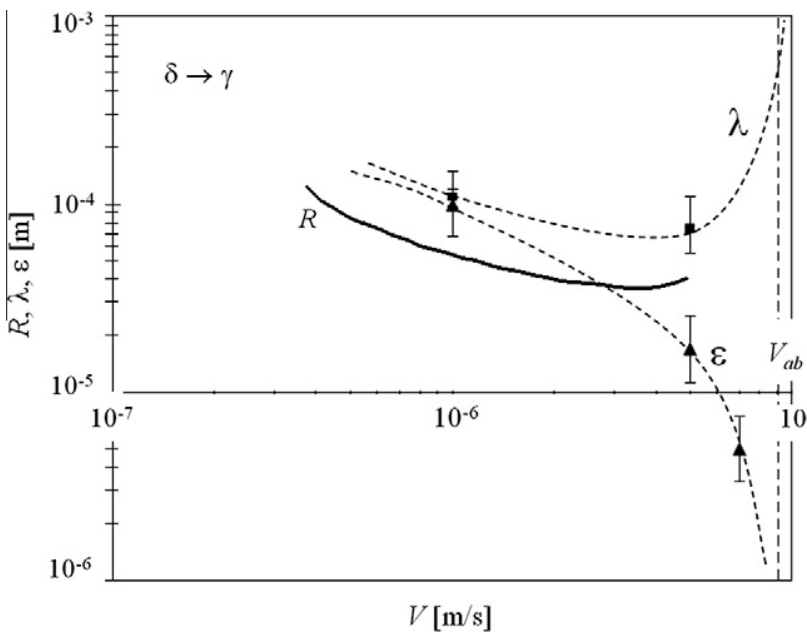

Fig. 5. Experimental cell tip spacing $\lambda$ and amplitude $\varepsilon$ vs $V$ for $\delta / \gamma$ transformation (interrupted curves). Cell tip radii $(R)$ were obtained with the I-MS model $[10,18]$ (continuous curve).

\section{Discussion}

\subsection{Solidification}

The experimental conditions were chosen such as to ensure plane front solidification below constitutional supercooling $V_{\text {cs }}$
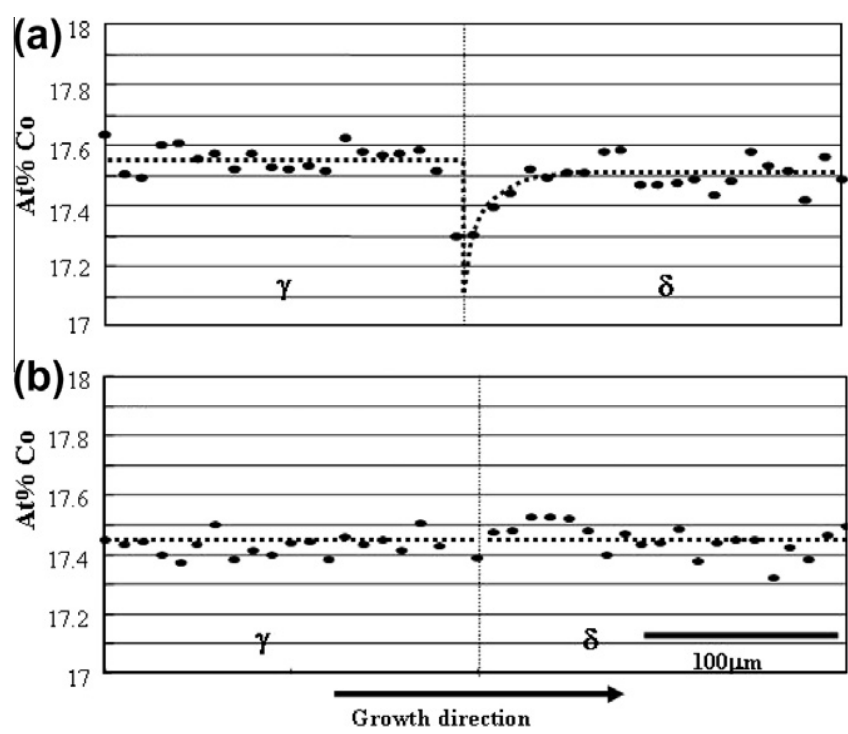

Fig. 6. Composition distribution across the $\delta / \gamma$ interface along the growth direction in the center of the specimen for: (a) $V=1 \mu \mathrm{m} \mathrm{s}^{-1}$ and (b) $V=10 \mu \mathrm{m} \mathrm{s}^{-1}$. Dotted vertical lines represent phase boundaries from micrographs. Owing to a lack of spatial resolution of the microprobe, the boundary layer in (b) is not visible.

$V_{\mathrm{cs}}=G D / \Delta T_{\mathrm{o}}$

where $G$ is the temperature gradient at the interface, $D$ is the solute diffusion coefficient in liquid, $\Delta T_{\mathrm{o}}$ is the equilibrium 
liquidus-solidus temperature interval. Using the properties in Table 2, the limit of constitutional undercooling for solidification of $\delta$ is obtained as $V_{\mathrm{cs}}=24.6 \mu \mathrm{m} \mathrm{s}^{-1}$. This is consistent with the observation of $\delta$ plane front growth in all specimens. Steady-state plane front solidification leads to a homogeneous solid, which is a microstructure-free substrate for subsequent transformation. The concentration measurements (Fig. 6) show that solidification of the specimen at $1 \mu \mathrm{m} \mathrm{s}^{-1}$ forms $\delta$ with 17.52 at. $\%$ Co.

Microstructure selection for solidification may be discussed with the aid of the concept of the interface response (IR) $[10,18]$. This model allows calculation of the solidification front temperature as a function of the interface velocity for different growth morphologies (Fig. 7, upper solid curve): At a low velocity and steady-state, the $S / L$ interface grows with planar morphology at the solidus temperature $T_{\mathrm{s}}$. Beyond the limit of constitutional undercooling $V_{\mathrm{cs}}$ the interface becomes cellular, and the interface temperature rises with $V$ according to $\left.T_{\mathrm{i}}=T_{1}-G D / V[19,20]\right)$. The temperature and radius of the dendrite tip in the medium velocity range can be calculated with the I-MS model $[8,9]$. The tip temperature decreases with increasing $V[18]$. The dendrites become finer with velocity and then turn into cells. Finally, above the critical velocity $V_{\mathrm{ab}}$, where the diffusion at the interface becomes strongly localized, plane front growth reappears [11]. This phenomenon is called absolute stability. $V_{\mathrm{ab}}$ for solidification corresponds approximately to the high $V$ minimum of the upper $T_{\mathrm{i}}$ curve in Fig. 7 and can be expressed as:

$V_{\mathrm{ab}}=D \Delta T_{\mathrm{o}} / k \Gamma$

where $k$ is the distribution coefficient, and $\Gamma$ is the Gibbs Thomson coefficient [9]. If $\Delta T_{\mathrm{o}}$ is large, an oscillatory instability of the plane front appears at $V_{\mathrm{ab}}$. In systems with small $\Delta T_{\mathrm{o}}$ values and large $G$ values, dendrites do not develop, and oscillatory instabilities are not formed. This is the case in the present alloy with small $\Delta T_{\mathrm{o}}$.

In rapid solidification of the order of meters per second, local equilibrium is lost and, owing to solute trapping, the solidus (=IR of the plane front in steady-state) rises and approaches $T_{\mathrm{o}}$, the temperature of equal free enthalpies. Finally, the atom attachment effect takes over at very high velocities and makes the interface temperature drop. This

Table 2

Parameters used for the calculations of the $L / \delta$ and $\delta / \gamma$ interface response in $\mathrm{Fe}-17.5$ at. $\%$ Co.

\begin{tabular}{llll}
\hline Parameter & & $L \rightarrow \delta$ & $\delta \rightarrow \gamma$ \\
\hline$T_{1}, T_{\delta}$ & $(\mathrm{K})$ & 1809.30 & 1690.10 \\
$m_{\mathrm{L}}, m_{\delta}$ & $(\mathrm{K} /$ at. $\%)$ & -1.81 & 4.74 \\
$k$ & & 0.9077 & 1.023 \\
$C_{0}$ & $($ at. $\%)$ & 17.5 & 17.5 \\
$D$ & $\left(\mathrm{~m}^{2} \mathrm{~s}^{-1}\right)$ & $4.4 \times 10^{-9}$ & $1.65 \times 10^{-11}$ \\
$\Gamma$ & $(\mathrm{K} \mathrm{m})$ & $2 \times 10^{-7}$ & $3.21 \times 10^{-6}$ \\
$a_{0}$ & $(\mathrm{~m})$ & $5 \times 10^{-9}$ & $2 \times 10^{-9}$ \\
$V_{0}$ & $\left(\mathrm{~m} \mathrm{~s}^{-1}\right)$ & 2000 & 5000 \\
$\Delta S_{\mathrm{tr}}$ & $\left(\mathrm{J} \mathrm{mol}^{-1} \mathrm{~K}^{-1}\right)$ & 8 & 1.124 \\
$G$ & $\left(\mathrm{~K} \mathrm{~m}^{-1}\right)$ & $1.8 \times 10^{4}$ & $1.8 \times 10^{4}$ \\
\hline
\end{tabular}

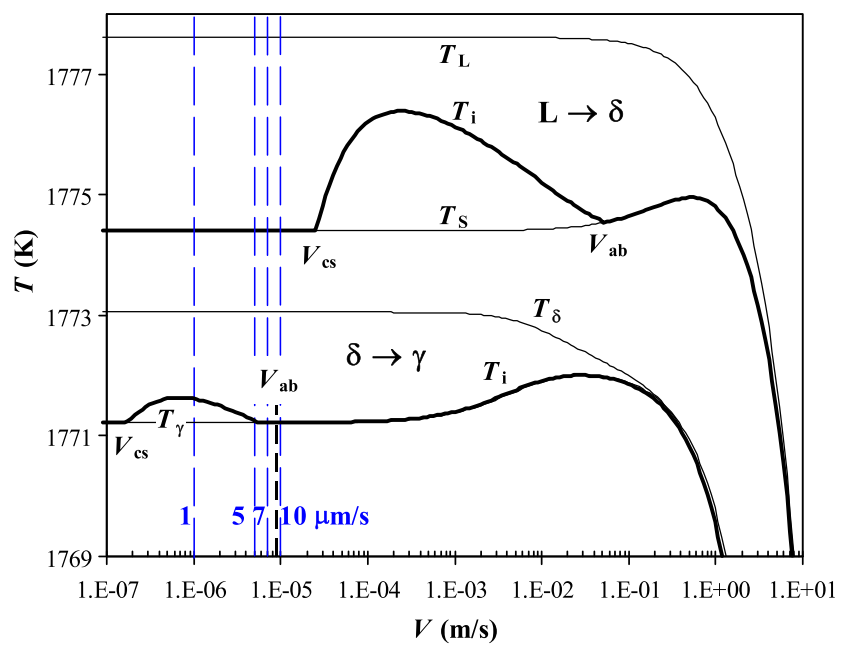

Fig. 7. Calculated temperature of the $L / \delta$ and $\delta / \gamma$ interface as a function of the growth velocity in directional transformation of $\mathrm{Fe}-17.5$ at.\% $\mathrm{Co}$. The thin lines correspond to the liquidus, solidus and solvus temperatures corrected for the effect of solute trapping and atom attachment kinetics. The thick curves represent the interface temperature $T_{\mathrm{i}} . V_{\mathrm{cs}}$ and $V_{\mathrm{ab}}$ are the constitutional supercoooling limit for planar growth and the critical velocity for absolute stability, respectively. The interrupted vertical lines indicate the velocities studied.

leads to the characteristic shape of the IR with two maxima, as shown in Fig. 7. Details of this model may be found elsewhere $[5,18]$.

\subsection{Solid-state transformation}

This theoretical approach developed for solidification microstructure selection [10] may also be applied to solidstate transformations if the interface is sufficiently mobile (incoherent) and stress effects are small [5,7]. At the very high temperatures of the present phase transformation, both conditions seem to be fulfilled. Furthermore, the diffusion coefficient of Co in $\delta$ is $\sim 24$ times larger than in $\gamma$, and therefore volume diffusion in the parent phase is the controlling transport process, as is the case in solidification. Isotropic diffusion behavior of the parent $\delta$ phase is assumed. With this in mind, steady-state solidification theory is applied to the $\delta-\gamma$ solid-state transformation.

A composition invariant product phase can be obtained without solute trapping by steady-state plane front transformation [21]. The steady-state is established after an initial transient, the typical length $L_{\mathrm{i}}$ of which can be estimated [9]

$L_{\mathrm{i}}=4 D / V k$

with $D$ the solute diffusion coefficient in the parent phase. Table 3 shows the relevant values for this study, i.e., for slow directional solid-state transformation, for slow solidification and for a typical massive transformation rate during quenching. It is interesting to see that, in heat treatment and with an interface rate of $1 \mathrm{~mm} \mathrm{~s}^{-1}$, a steady-state is obtained over the extremely short distance of $65 \mathrm{~nm}$. For the growth of a $10-\mu \mathrm{m}$-thick layer of massive phase, the time 
Table 3

Initial transients and $D / V$ values for various transformations.

\begin{tabular}{llll}
\hline & $\begin{array}{l}\text { Low } V \text { directional } \\
\delta-\gamma \text { transformation }\end{array}$ & $\begin{array}{l}\text { Low } V \text { directional } \\
\text { solidification }\end{array}$ & $\begin{array}{l}\text { Quenching, rapid } \\
\delta-\gamma \text { transformation }\end{array}$ \\
\hline$V\left(\mathrm{~m} \mathrm{~s}^{-1}\right)$ & $1 \times 10^{-6}$ & $1 \times 10^{-6}$ & $1 \times 10^{-3}$ \\
$D\left(\mathrm{~m}^{2} \mathrm{~s}^{-1}\right)$ & $1.65 \times 10^{-11}$ & $4.4 \times 10^{-9}$ & $1.65 \times 10^{-11}$ \\
$L_{\mathrm{i}}(\mathrm{m})$ & $65 \times 10^{-6}$ & $19 \times 10^{-3}$ & $65 \times 10^{-9}$ \\
$D / V(\mathrm{~m})$ & $16.5 \times 10^{-6}$ & $4.4 \times 10^{-3}$ & $16.5 \times 10^{-9}$ \\
\hline
\end{tabular}

required under these conditions is $10 \mathrm{~ms}$. Table 3 indicates that in these experiments a steady-state was achieved under all conditions.

The IR for the solid-state $\delta / \gamma$ transformation is also given in Fig. 7 using the material properties from Table 2 (lower solid curve). This IR ( $T_{\mathrm{i}}$ curve) has a form similar to that in solidification, also showing two maxima. The limit of constitutional supercooling $V_{\text {cs }}$ and absolute stability $V_{\mathrm{ab}}$ are calculated to be $0.16 \mu \mathrm{m} \mathrm{s}^{-1}$ and $9.2 \mu \mathrm{m} \mathrm{s}^{-1}$, respectively. The growth velocities used in the experiments for the solid-state transformation are also indicated in Fig. 7. Owing to the extremely narrow two-phase range of the alloy, $\gamma$ develops no dendrites (only cells), and banding does not occur. At $1 \mu \mathrm{m} \mathrm{s}^{-1}$, the $\delta$-phase with $C=17.52$ at.\% Co transforms into cellular $\gamma$ with a composition of 17.55 at.\% (Fig. 6a). The slightly higher concentration of the cell centers is expected as their interface temperature is above $T_{\gamma}$ (Fig. 7). The experimental boundary layer thickness from Fig. $6 \mathrm{a}$ is $35 \mu \mathrm{m}$, which compares well with the calculated value of $2 D / V=33 \mu \mathrm{m}$ (Table 3 ).

The I-MS model was also used to calculate the dendrite/ cell tip radius. In Fig. 5, the calculated tip radii $R$ (continuous line) are superimposed onto the measured spacings $\lambda$, and show a similar tendency. As shown in Fig. 4, flat cells are observed at $7 \mu \mathrm{m} \mathrm{s}^{-1}$, while the plane front $\gamma$ was clearly seen at $10 \mu \mathrm{m} \mathrm{s}^{-1}$. Therefore, the experimentally determined $V_{\mathrm{ab}}$ value is between these two growth rates. This result agrees well with the calculated value of $V_{\mathrm{ab}}=9.2 \mu \mathrm{m} \mathrm{s}^{-1}$. This observation supports the conclusion that the planar $\delta / \gamma$ solid-state transformation interface has reached absolute stability beyond this velocity.

The $D / V$ value for $V=10 \mu \mathrm{m} \mathrm{s}^{-1}$ is so thin $(1.65 \mu \mathrm{m})$ that a spike cannot be detected with the EPMA technique. From the theoretical model, however, it is concluded that a spike exists and that composition invariance is obtained through steady-state plane front growth.

Fig. 8 shows the evolution of the interface temperature (Fig. 8a) and the interface compositions (Fig. 8b) as a function of velocity. Just beyond $V_{\mathrm{ab}}$, the plane front grows at $T_{\gamma}$ under conditions which are close to local equilibrium (A). At a higher rate, the interface temperature rises (B) and reaches a maximum which corresponds to a combined effect of solute trapping and attachment kinetics. Further acceleration lowers the interface temperature owing to attachment kinetics (C). In this case, there is also substantial solute trapping. Only at a very high growth rate of several $10 \mathrm{~cm}$ per second is the condition of complete solute trapping achieved (D). One sees that the interface temperature $T_{\mathrm{i}}$ evolves from $T_{\gamma}$, rises to but never reaches $T_{\mathrm{o}}$ before it falls below $T_{\gamma}$.

Heat flow calculations were performed in order to evaluate the typical interface velocity that can be expected dur-

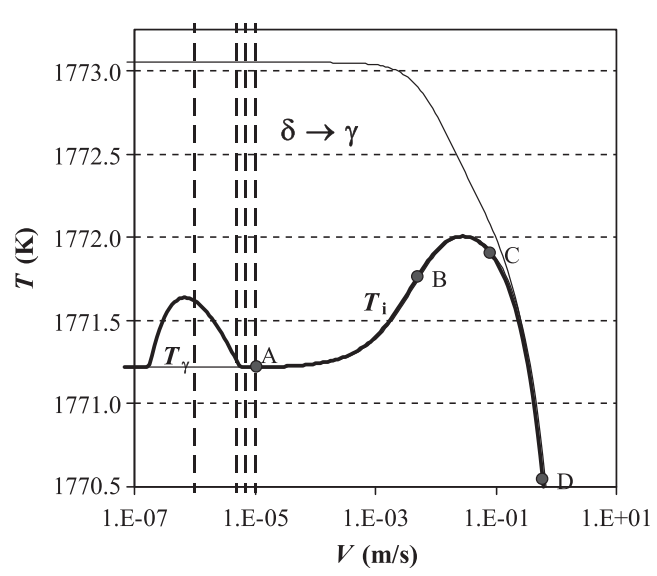

(a)

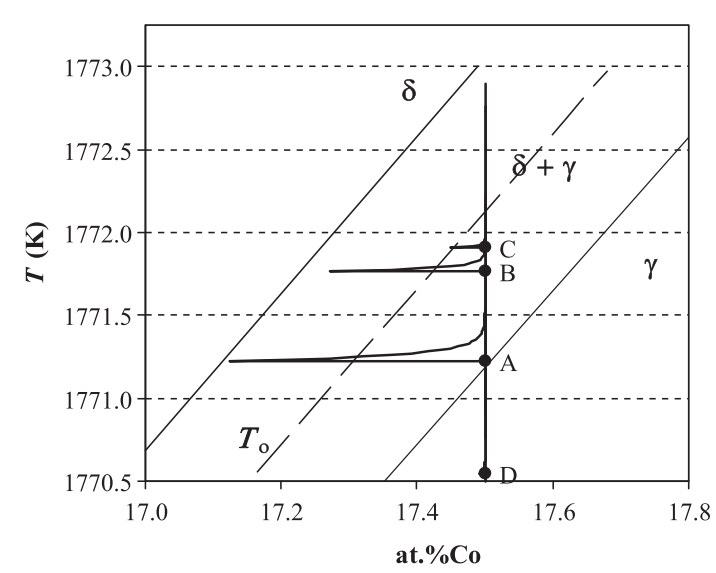

(b)

Fig. 8. (a) $\delta / \gamma$ Interface temperature and (b) Phase diagram and concentration profiles in front of the interface for four different velocities (A-D), all leading to massive transformation. A concentration spike at local equilibrium is formed in front of the interface when absolute stability is reached at A. Even at a maximum velocity of centimeters per second only partial solute trapping is predicted, while at point $\mathrm{D}$ at $V=1 \mathrm{~m} \mathrm{~s}$ complete solute trapping takes place. The latter velocity, however, might be too high for solid-state transformations. 
ing quenching in an industrial heat treatment process. The calculations were based on the resolution of the heat conservation equation in a one-dimensional domain divided into two regions corresponding to $\gamma$ and $\delta$. A very thin layer of $\delta$ was initiated at the left border of the domain at the beginning of the calculation. A heat transfer coefficient was applied on the left border, whereas a zero-flux boundary condition was used at the right extremity (symmetry plane). The position of the $\delta / \gamma$ interface was updated at every time-step, using the interface response of Fig. 7 evaluated for the local temperature.

Fig. 9 shows the thermal gradient and the interface velocity as a function of the position of the interface $x^{*}$, obtained for a 2-mm-thick steel plate and two different values of the heat transfer coefficient $h$. With $h=$ $200 \mathrm{~W}\left(\mathrm{~m}^{2} \mathrm{~K}\right)^{-1}$ (Fig. 9a), the interface velocity is of the order of $1.5 \mathrm{~mm} \mathrm{~s}^{-1}$. The first increase is associated with the transient regime for the interface to reach the undercooling corresponding to the local isotherm velocity. Then, the interface follows the isotherms, which move more
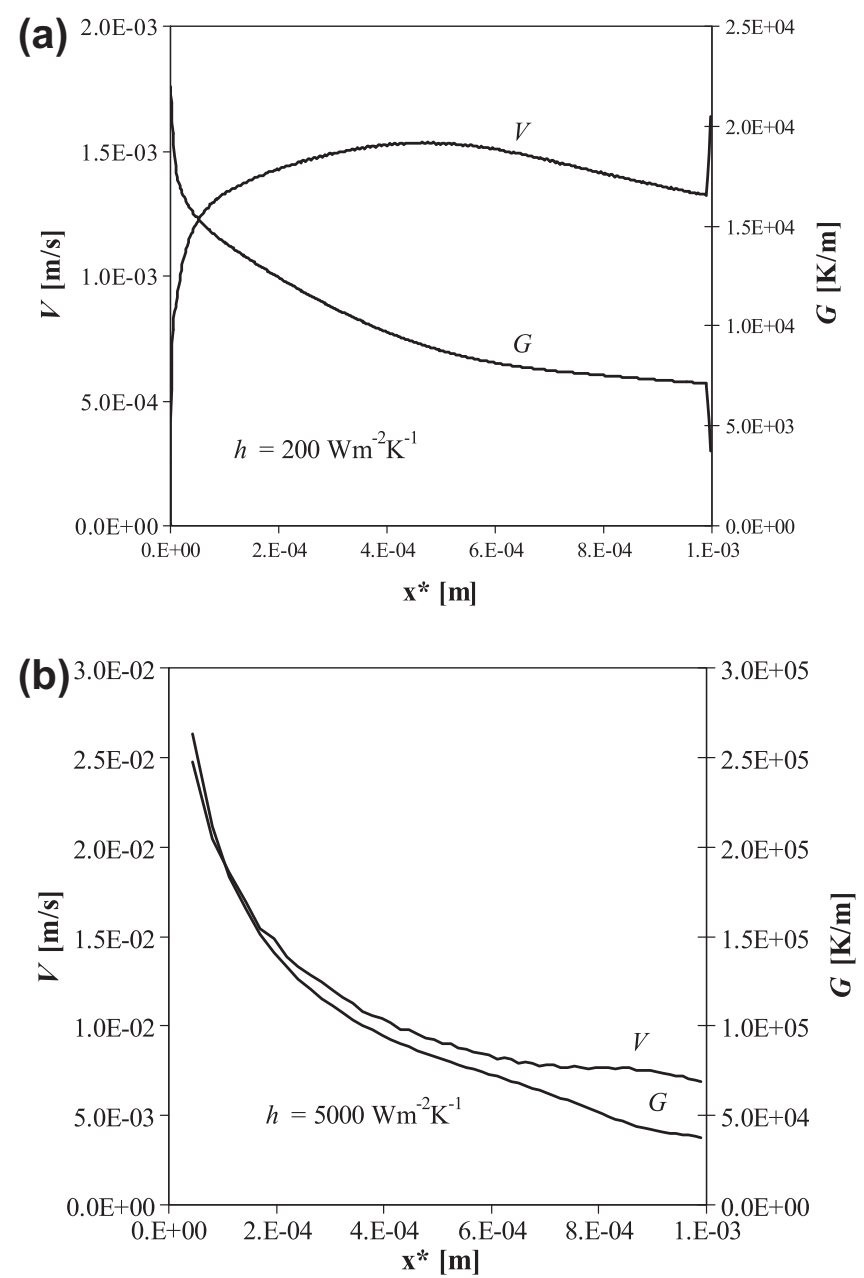

Fig. 9. Interface velocities and temperature gradients as a function of distance for a 2-mm-thick steel plate calculated with a 1-dimensional heat flow model. Two extreme heat transfer coefficients of quenching (a) For air, $h=200 \mathrm{~W}\left(\mathrm{~m}^{2} \mathrm{~K}\right)^{-1}$, and (b) For brine, $h=5000 \mathrm{~W}\left(\mathrm{~m}^{2} \mathrm{~K}\right)^{-1}$, were used. slowly deeper in the plate. In the case of the higher heat transfer coefficient of $5000 \mathrm{~W}\left(\mathrm{~m}^{2} \mathrm{~K}\right)^{-1}$ (Fig. 9b), the transient regime is much shorter due to steeper thermal gradients and the interface velocity is more than 10 times higher than that for the lower value, i.e., the interface moves at $1-2.5 \mathrm{~cm} \mathrm{~s}^{-1}$.

In Fig. 10, these velocities are superimposed onto the IR curve of the solid-state transformation of Fig. 7. This figure clearly indicates that massive transformation under practical conditions of heat treatment and quenching may happen through steady-state plane front growth with only limited solute trapping. As this figure shows, a heat transfer coefficient of $200 \mathrm{~W}\left(\mathrm{~m}^{2} \mathrm{~K}\right)^{-1}$ produces transformation rates which are close to local equilibrium. Transformations with a high heat transfer take place in-between local equilibrium and complete solute trapping. In these cases, it is the non-equilibrium solvus which forms the reference temperature for the transformation.

These calculations were performed assuming the interface velocity to be equal to that of the isotherms. The interface velocity may in reality be higher if repeated nucleation events are required to propagate the massive microstructure deeper in the material (equiaxed transformation). In the $\mathrm{Fe}-$ Co system, the interface velocity must, however, be $>10 \mathrm{~cm} \mathrm{~s}^{-1}$ to reach the regime of complete solute trapping.

\section{Conclusion}

In analogy to standard Bridgman-type directional solidification, directional $\delta-\gamma$ solid-state transformation experiments have been undertaken in $\mathrm{Fe}-\mathrm{Co}$ alloy. Directional growth experiments, although seldom used, are very useful for examining solid-state transformation phenomena. Good control of the interface rate and the temperature gradient, and the quantitative measurement of the interface temperature are the main advantages of this technique.

With increasing growth rate in the range $1-10 \mu \mathrm{m} \mathrm{s}^{-1}$, transition from a cellular to a planar interface structure was observed in $\mathrm{Fe}-17.5$ at.\% Co. Volume diffusion-controlled I-MS theory was applied for the interpretation of the microstructures observed in the solid state close to the melting point. It is concluded that plane front stabilization is the result of absolute stability, a phenomenon which was predicted theoretically by Mullins and Sekerka in 1964 [11]. To the knowledge of the authors, it is the first time that a quenched absolutely stable plane front has been shown experimentally.

Owing to the small diffusion coefficients in solids, highvelocity phenomena such as absolute stability which have met substantial experimental difficulties in liquid-solid transformations may be favorably investigated in the solid state.

In the steady-state, plane front growth produces a composition-invariant crystal with a narrow concentration spike at the moving boundary. A massive transformation product may therefore form with no or partial solute trapping. In this case, the non-equilibrium solvus and not $T_{\mathrm{o}}$ is 


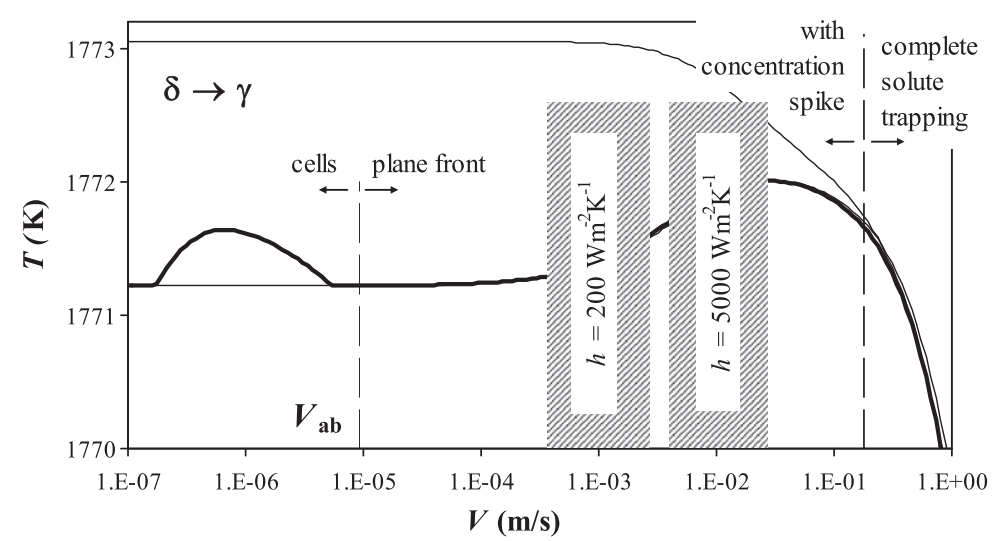

Fig. 10. Superposition of the range of interface velocities during quenching (from Fig. 9) onto the interface response (interface temperature vs velocity) curve for $\delta / \gamma$ transformation (lower solid curve of Fig. 7). For quenching in brine $\left(h=5000 \mathrm{~W}\left(\mathrm{~m}^{2} \mathrm{~K}\right)^{-1}\right)$, a massive transformation is predicted to take place at velocities which are far from complete solute trapping. Therefore, a thin concentration spike accompanies this transformation.

the relevant reference temperature. This is even true for the case of quenched specimens, where interface displacement rates can reach several centimeters per second. Much higher velocities are needed to reach complete solute trapping. This conclusion does not necessarily apply to systems exhibiting a massive transformation at lower temperatures.

\section{Acknowledgments}

The authors appreciate helpful collaboration of $\mathrm{M}$. Lima, J. Stramke and B. Neal, EPFL.

\section{References}

[1] Massalski TB. Phase transformations. Metals Park (OH): ASM; 1970. p. 433.

[2] Perepezko JH. Metall Trans 1984;15A:437.

[3] Borgenstam A, Hillert M. Acta Mater 2000;48:2765.

[4] Zurob HS, Hutchinson CR, Bréchet Y, Seyedrezai H, Purdy GR. Acta Mater 2009;57:2781.

[5] Vandyoussefi M, Kerr HW, Kurz W. Acta Mater 1997;45:4093.
[6] Kurz W, Lima M. In: Koiwa M, Otsuka K, Miyazaki T, editors. International conference on solid-solid phase transformations ' 99 (JIMIC-3). The Japan Institute of Metals; 1999. p. 1581.

[7] Lima M, Kurz W. Metall Mater Trans A 2002;33:2337.

[8] Kurz W, Giovanola B, Trivedi R. Acta Metall 1986;34:823.

[9] Kurz W, Fisher DJ. Fundamentals of solidification. Zurich, Switzerland: Trans Tech Publications Ltd; 1998

[10] Kurz W. Adv Eng Mater 2001;3:443.

[11] Mullins WW, Sekerka RF. J Appl Phys 1964;35:444.

[12] Kurz W, Trivedi R. Metall Mater Trans A 1996;27:625.

[13] Clayton DN, Hellawell A. Solidification processing 1987. London: The Institute of Metals; 1987. p. 156.

[14] Trivedi R, Sekhar JA, Seetharaman V. Metall Trans A 1989;20:769.

[15] Ludwig A, Kurz W. Acta Mater 1996;44:3643.

[16] Sumida M, Kurz W. Z Metallkd/Mater Res Adv Tech 2002;93:1154.

[17] Dobler S, Lo TS, Plapp M, Karma A, Kurz W. Acta Mater 2004;52:2795.

[18] Trivedi R, Kurz W. Int Mater Rev 1994;39:49.

[19] Bower TF, Brody HD, Flemings MC. Trans Metall Soc AIME 1966;236:625.

[20] Burden MH, Hunt JD. J Cryst Growth 1974;22:109.

[21] Hillert M. The mechanism of phase transformations. Crystalline solids. London: The Institute of Metals; 1969. 